\title{
Ectopic biomineralization in kidney stone formers compared to non-stone formers
}

\author{
Adrian M. Fernandez ${ }^{1}$, Benjamin A. Sherer ${ }^{1}$, Stuart A. Gansky ${ }^{2}$, Jorge D. Mena ${ }^{1}$, Sudarshan Srirangapatanam ${ }^{1}$, \\ Scott V. Wiener ${ }^{1}$, Thomas Chi ${ }^{1}$, Sunita P. $\mathbf{H o}^{2}$, Marshall L. Stoller ${ }^{1}$ \\ ${ }^{1}$ Department of Urology, University of California San Francisco, San Francisco, CA, USA; ${ }^{2}$ Department of Preventative and Restorative Dentistry, \\ University of California San Francisco, San Francisco, CA, USA \\ Contributions: (I) Conception and design: AM Fernandez, BA Sherer, SP Ho, ML Stoller; (II) Administrative support: JD Mena, S Srirangapatanam, \\ SV Wiener; (III) Provision of study materials or patients: SP Ho, T Chi, ML Stoller; (IV) Collection and assembly of data: AM Fernandez, BA \\ Sherer, JD Mena, S Srirangapatanam, SV Wiener; (V) Data analysis and interpretation: AM Fernandez, BA Sherer, SA Gansky, JD Mena, S \\ Srirangapatanam, SP Ho, ML Stoller; (VI) Manuscript writing: All Authors; (VII) Final approval of manuscript: All authors. \\ Correspondence to: Sunita P. Ho, PhD. Department of Preventative and Restorative Dentistry, University of California San Francisco, 513 Parnassus \\ Ave, San Francisco, CA 94143, USA. Email: Sunita.Ho@ucsf.edu; Marshall L. Stoller, MD. Department of Urology, University of California, San \\ Francisco, 400 Parnassus Ave, San Francisco, CA 94143, USA. Email: Marshall.Stoller@ucsf.edu.
}

Background: Kidney stone formers ( $\mathrm{SFs}$ ) are at increased risk of stroke, myocardial infarction, and atherosclerosis of the carotid and coronary arteries. These cardiovascular and urologic pathologies can result from ectopic biomineral deposition. The objectives of this study are: (I) to evaluate risk factors for ectopic biomineralization, and (II) to characterize the overall burden of ectopic minerals in known SFs compared to non-stone formers (NSFs) matched for these risk factors.

Methods: Presence and quantity of biominerals at eight anatomic locations (abdominal aorta, common iliac arteries, pelvic veins, prostate or uterus, mesentery, pancreas, and spleen) were determined in a case control study by retrospective analysis of clinical non-contrast computed tomography scans obtained from 190 SFs and 190 gender- and age-matched NSFs (renal transplant donors). Predictors of biomineralization were determined using negative binomial regression. A subgroup of $140 \mathrm{SF}$ s and $140 \mathrm{NSF}$ s were matched for risk factors for systemic biomineralization, and mineralization was compared between these matched SFs and NSFs using ordinal logistic regression.

Results: Hypertension, hyperlipidemia, diabetes mellitus, and smoking were more common amongst SFs. Risk factors for increased systemic biomineralization included history of nephrolithiasis, male gender, older age, and history of hyperlipidemia. When controlling for these comorbidities, SFs had significantly increased biomineralization systemically and at the abdominal aorta, iliac arteries, prostate, mesentery, pancreas, and spleen compared to NSFs.

Conclusions: The current study provides evidence that SFs are at increased risk of biomineralization systemically, independent of common risk factors of atherosclerosis.

Keywords: Atherosclerosis; biomineralization; computed tomography; nephrolithiasis

Submitted Dec 28, 2019. Accepted for publication Jul 30, 2020.

doi: $10.21037 /$ tau-19-927

View this article at: http://dx.doi.org/10.21037/tau-19-927

\section{Introduction}

Pathologic minerals continue to be observed in various locations of the human body. Numerous population-based studies have confirmed that patients who form kidney stones (a type of pathologic mineral) often have systemic diseases including obesity (1-4), diabetes mellitus (DM) $(4,5)$, and hypertension (HTN) $(6,7)$ that portend risk for calcium-based vascular pathology. Kidney stone formers 
(SFs) have an increased relative risk of coronary artery mineralization (8), abdominal aortic mineralization (9), coronary artery disease $(10)$, myocardial infarction $(11,12)$, carotid atherosclerosis (13) and stroke (14). The association between biomineralization in the renal tissue (nephrolithiasis) and at non-renal, non-osseous sites is poorly understood. The objectives of this study are two-fold: (I) to evaluate risk factors for ectopic biomineralization at multiple locations/ organs; and (II) to characterize the overall burden of ectopic mineral deposition in known kidney SFs compared to nonstone formers (NSFs) matched for recognized risk factors. The hypothesis is that cardiovascular risk factors including elevated body mass index (BMI), DM, HTN, hyperlipidemia (HLD), hyperparathyroidism (HPTH) and smoking demonstrate a strong association with ectopic mineralization and that SFs will have increased pathologic mineralization at multiple anatomic sites compared to matched controls. We present the following article in accordance with the STROBE reporting checklist (available at http://dx.doi. org/10.21037/tau-19-927).

\section{Methods}

The presence and quantity of biominerals at eight nonrenal anatomic locations were determined by a retrospective analysis of clinical non-contrast $\mathrm{X}$-ray computed tomography (CT) scans (GE Healthcare, $2.5 \mathrm{~mm}$ continuous slices, approximately $20 \mathrm{mGy}$ per scan) of the abdomen/ pelvis obtained from known SFs and a matched cohort of NSFs (renal transplant donors). Non-renal, non-osseous sites evaluated included the abdominal aorta, common iliac arteries, pelvic veins (phleboliths), prostate or uterus, mesentery, pancreas (pancreatic vessels), and spleen (splenic vessels). These anatomical sites were chosen because they were captured consistently within the field of view of the CT of the abdomen and pelvis, a routine diagnostic imaging test used for evaluating both SFs and NSFs.

\section{Patient selection}

Institutional review board (IRB) approval was obtained to review CT scans of previously established SF and renal donor databases (IRB\# 16-21330), and the study was conformed to the provisions of the Declaration of Helsinki (as revised in 2013). The NSF control group consisted of kidney transplant donors with CT scans obtained prior to organ donation between 2012 and 2016. All patients provided a consent to review de-identified images and medical records for research purposes. Only patients with a non-contrast CT of the abdomen and pelvis available for review within our institutional electronic imaging database were included for analyses. Renal transplant donors with documented history of nephrolithiasis or obvious renal stones demonstrated in CT scans were excluded from the final analyses. One hundred ninety $(n=190)$ kidney transplant donors from the donor database met inclusion criteria for the study. One-hundred ninety $(n=190)$ SF experimental subjects were subsequently selected from a database of patients with known nephrolithiasis who underwent operative treatment and comprehensive stone analytics at our tertiary care center between 2012 and 2017. These 190 SFs were the most recently treated patients in the database with similar marginal balance on age and gender to NSFs (Table 1). These subjects constitute the General Sample ( $\mathrm{n}=380)$.

Comorbidities (i.e., elevated BMI, DM, HTN, HLD, HPTH, and smoking history) that have been previously implicated in stone and vascular diseases were evaluated in the General Sample. For all SFs, stone composition, stone size, stone location, stone treatment modality, history of recurrent nephrolithiasis, and history of infection stones were recorded. A disparity between comorbidities was noted in the general sample size of 380 patients that included 190 SFs and 190 NSFs (Table 1). To establish equal baseline risk factor distribution between $\mathrm{SF}$ and NSFs, a subgroup of 280 patients ( $140 \mathrm{SFs}$ and $140 \mathrm{NSF}$ ) matched for these comorbidities were selected for further analysis from the General Sample population (Table 2). The selection of comorbidity-matched patients in the Matched Sample $(n=280)$ was based on including SFs with the greatest burden of comorbidities and NSFs with the least burden of comorbidities from a sample of original 380 in order to achieve similar marginal balance on comorbidities, age, and gender.

\section{Review of CT scans and data acquisition}

Non-contrast CT scans of the abdomen/pelvis of all patients were independently reviewed by a single fellowship trained endourologist (BS, one of the co-authors) for the presence and quantity of mineralization at the eight aforementioned non-renal sites. Review of CT scans could not be blinded given the presence of renal calculi on CT scans of SFs. At all anatomical sites in each patient, mineral deposits were measured objectively in three dimensions. These minerals were scored based on total volume of minerals and the data 
Table 1 Patient characteristics

\begin{tabular}{lccc}
\hline Characteristic & Stone formers $(\mathrm{n}=190)$ & Non-stone formers $(\mathrm{n}=190)$ & $\mathrm{P}$ value \\
\hline Female, $\mathrm{n}[\%]$ & $105[55]$ & $105[55]$ & 1 \\
Male, $\mathrm{n}[\%]$ & $85[45]$ & $85[45]$ & $49 \pm 0.9$ \\
Age at CT & $48.5 \pm 0.7$ & $27.3 \pm 0.3$ & 0.728 \\
Body mass index & $30.6 \pm 0.7$ & 0 & $<0.001$ \\
Diabetes mellitus, $\mathrm{n}[\%]$ & $27[14]$ & $13[7]$ & $<0.001$ \\
Hypertension, $\mathrm{n}[\%]$ & $61[32]$ & $27[14]$ & $<0.001$ \\
Hyperlipidemia, $\mathrm{n}[\%]$ & $59[31]$ & $43[23]$ & 0.001 \\
Smoking, $\mathrm{n}$ [\%] & $51[27]$ & 0.405 & 0.317 \\
Hyperparathyroidism & 1 & 0 & $<$ \\
\hline
\end{tabular}

Table 2 Patient characteristics for comorbidity matched sample

\begin{tabular}{lccc}
\hline Characteristic & Stone formers $(\mathrm{n}=140)$ & Non-stone formers $(\mathrm{n}=140)$ & $\mathrm{P}$ value \\
\hline Female, $\mathrm{n}[\%]$ & $73[52]$ & $73[52]$ & 1 \\
Male, $\mathrm{n}[\%]$ & $67[48]$ & $67[48]$ & 1 \\
Age at CT & $46.2 \pm 1$ & $49.5 \pm 0.8$ & 0.011 \\
Body mass index & $29 \pm 0.7$ & $27.9 \pm 0.3$ & 0.172 \\
Diabetes mellitus, $\mathrm{n}[\%]$ & $1[1]$ & 0 & 1 \\
Hypertension, $\mathrm{n}[\%]$ & $22[16]$ & $13[9]$ & 0.148 \\
Hyperlipidemia, $\mathrm{n}[\%]$ & $26[19]$ & $27[19]$ & 1 \\
Smoking, $\mathrm{n}[\%]$ & $29[21]$ & $30[21]$ & 1 \\
Hyperparathyroidism & 1 & 0 & 0.316 \\
\hline
\end{tabular}

was presented as $0=$ none, $1=\leq 0.5 \mathrm{~cm}^{3}, 2=0.5$ to $1.0 \mathrm{~cm}^{3}$, $3=\geq 1.0 \mathrm{~cm}^{3}$ (Figure 1). The tiered severity scoring system was adopted from the methods used to compare coronary artery mineralization by Hsi et al., 2016 (8). Each anatomic site of interest was evaluated in axial, coronal, and sagittal planes and assigned a mineralization severity score (MSS) ranging $0-3$ based on calculated CT volume of visible mineralization. An overall mineralization score (OMS), which ranged 0 to 14 , was calculated for each study participant by summing the MSS across seven anatomic sites for each individual.

\section{Statistical analysis}

Demographics and comorbidities were compared between SFs and NSFs using Fisher's exact test for dichotomous characteristics and 2-sample t-test (with Satterthwaite unequal variance, if appropriate) for continuous characteristics to understand baseline differences between groups.

SF/NSF status, gender, age, BMI, history of DM, HTN, HLD, and smoking history were evaluated for their effect on mineralization severity (OMS) using negative binomial regression. Zero-inflated Poisson and zero-inflated negative binomial regression were evaluated as alternative models, but neither significantly increased model fit.

The MSS at each of the eight anatomic sites was compared between groups using ordinal logistic regression, adjusted for gender, age at time of CT acquisition, BMI, and comorbidities in SFs vs. NSFs ("Matched Sample", $\mathrm{n}=280$ ). The model's proportional odds assumption was assessed to ensure appropriateness. Due to the rarity of 

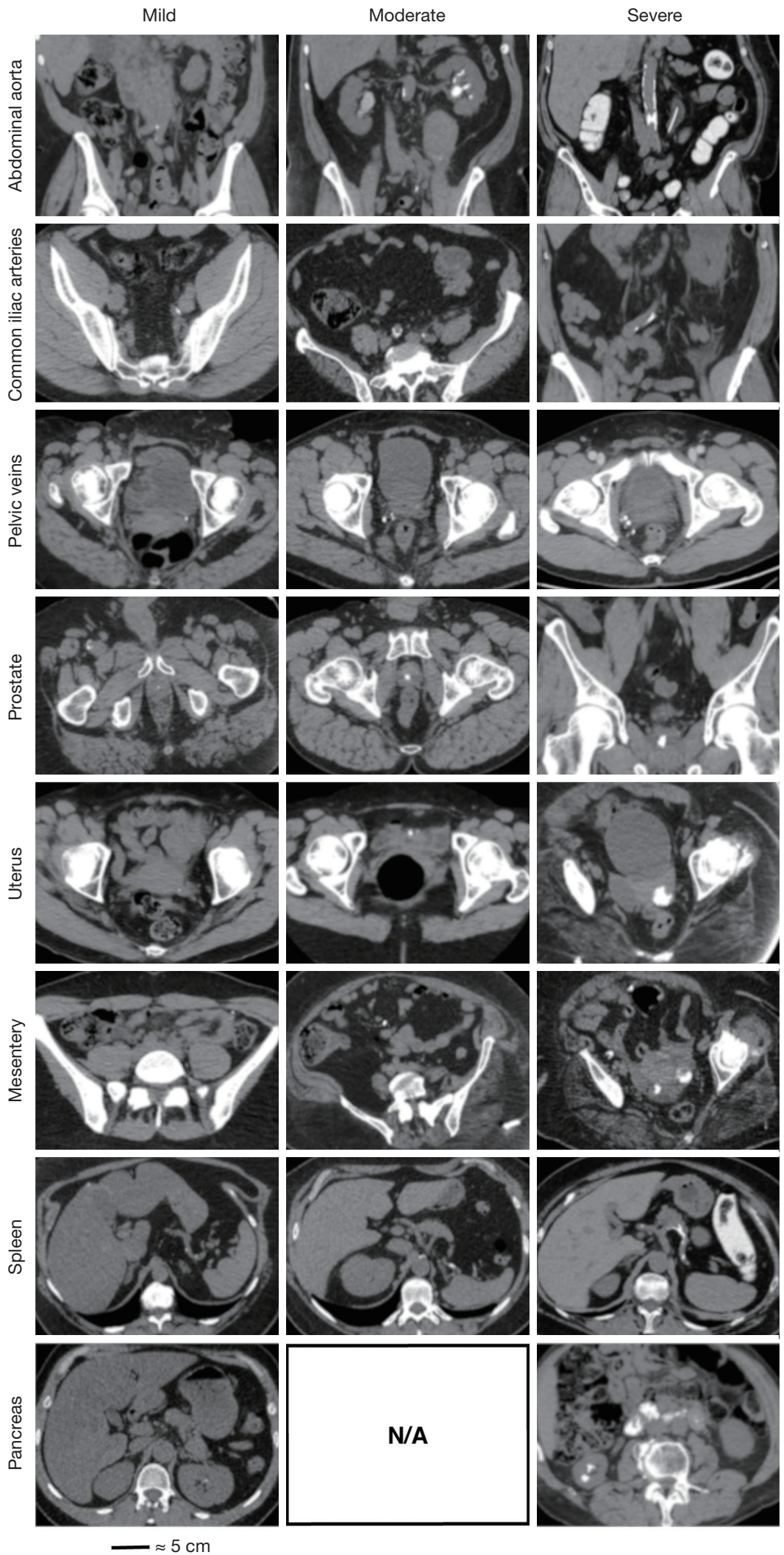

Figure 1 Measurement of ectopic mineralization. 
biominerals with higher scores in the pancreas and spleen, ordinal logistic regression was not appropriate, so binary logistic regression was used. The model was used to assess the likelihood of observing higher MSS in SFs compared to NSFs. Finally, a combined odds ratio for overall ectopic mineral burden was then calculated using weighted estimate with inverse variance weights.

\section{Results}

The General Sample contained 380 patients, with similar

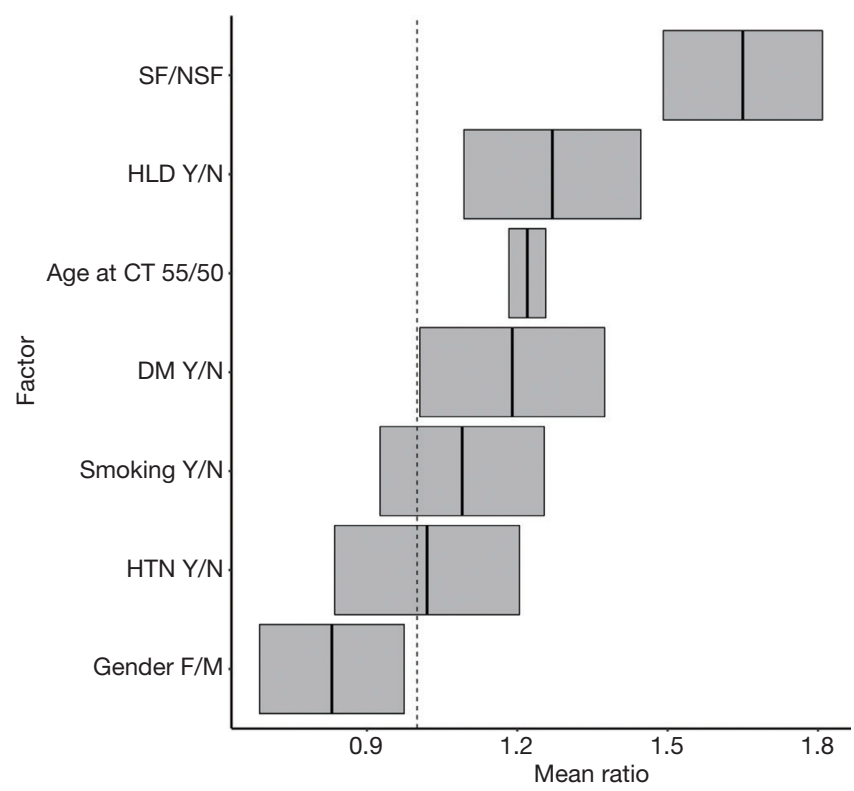

Figure 2 Predictors of calcification include nephrolithiasis, male gender, and hyperlipidemia. age and gender distributions. SFs had increased BMI and history of DM, HTN, and HLD (Table 1). All patients contained biominerals in multiple anatomical locations (Figure 1).

Negative binomial regression indicated that, on average, SFs had greater OMS than NSFs (SF/NSF OMS mean ratio=1.65, $\mathrm{P}<0.01)$. Other factors that significantly increased average OMS included gender (male $v$ s. female OMS mean ratio $=1.2, \mathrm{P}=0.01)$, age $(55 v s .50$ years old OMS mean ratio $=1.22, \mathrm{P}<0.01$ ) and history of HLD (OMS mean ratio $=1.27, \mathrm{P}<0.01)$. History of $\mathrm{DM}(\mathrm{OMS}$ mean ratio $=1.19, \mathrm{P}=0.12$ ), HTN $(\mathrm{OMS}$ mean ratio $=1.02$, $\mathrm{P}=0.80$ ), and smoking (OMS mean ratio $=1.09, \mathrm{P}=0.28$ ) showed no significant association with increased OMS (Figure 2).

With this understanding of comorbidities associated with increased OMS, a comorbidity-match sample (Matched Sample, n=280) of 140 SFs and 140 matched NSFs was selected, in which BMI, DM, HTN, HLD, hyperparathyroidism, and smoking status were not statistically different between SFs and NSFs (Table 2). Scores at each anatomical location was compared between SFs and NSFs in the General Sample and Matched Sample (Table 3, Figure 3). SFs had significantly increased likelihood of having higher MSS involving the abdominal aorta (OR 2.68, $\mathrm{P}<0.01)$, common iliac arteries (OR 2.92, $\mathrm{P}<0.01$ ), prostate (OR 3.26, $\mathrm{P}<0.01$ ), mesentery (OR 4.46, $\mathrm{P}<0.01$ ), pancreas (OR 9.99, $\mathrm{P}<0.01$ ), and spleen (OR 3.22, $\mathrm{P}<0.01)$. SFs also had greater mineralization severity overall (combined OR 2.28, $\mathrm{P}<0.01$ ).

Finally, 77 SFs and 85 NSFs with no history of DM, HTN, HLD, or smoking were compared with respect to

Table 3 Average mineralization severity scores (MSS)

\begin{tabular}{|c|c|c|c|c|c|c|}
\hline & \multicolumn{2}{|c|}{ General sample $(n=380)$} & $\mathrm{P}$ & \multicolumn{2}{|c|}{ Matched sample $(n=280)$} & $\mathrm{P}$ \\
\hline Aorta & 0.85 & 0.39 & $<0.01$ & 0.64 & 0.43 & 0.05 \\
\hline Iliac arteries & 0.84 & 0.37 & $<0.01$ & 0.64 & 0.38 & 0.02 \\
\hline Pelvic veins & 1.15 & 0.89 & 0.01 & 1.02 & 0.91 & 0.34 \\
\hline Uterus & 0.61 & 0.48 & 0.2 & 0.51 & 0.41 & 0.37 \\
\hline Mesentery/bowel & 0.24 & 0.05 & $<0.01$ & 0.2 & 0.05 & $<0.01$ \\
\hline Spleen & 0.21 & 0.07 & $<0.01$ & 0.12 & 0.08 & 0.39 \\
\hline Pancreas & 0.11 & 0.01 & $<0.01$ & 0.06 & 0.01 & 0.01 \\
\hline
\end{tabular}




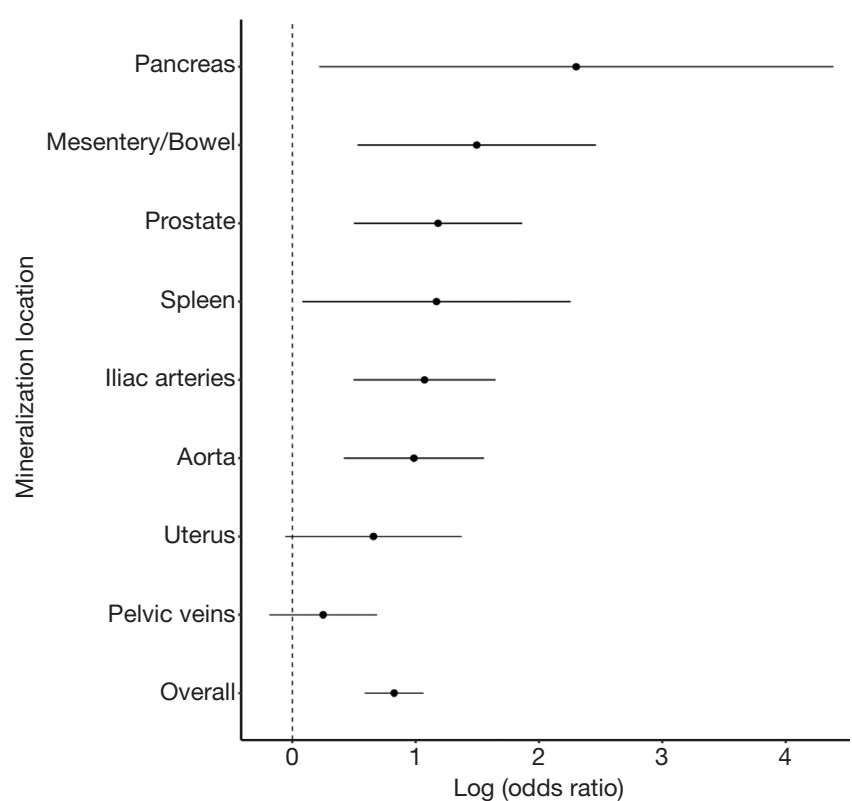

Figure $3 \mathrm{SF}$ s have increased calcification at many anatomic locations and overall.

mineral deposition across anatomic sites (Table S1). SFs had higher MSS at the abdominal aorta (OR 3.92, $\mathrm{P}<0.01)$, iliac arteries (OR 3.34, $\mathrm{P}<0.01$ ), and in the mesentery (OR 8.7, $\mathrm{P}<0.01)$ and lower MSS than NSFs at the prostate (OR 0.36, $\mathrm{P}<0.01)$ (Table S2).

\section{Discussion}

In a long-term effort to further understand the etiology of kidney stone formation, the relationship between nephrolithiasis, atherosclerotic risk factors, and systemic ectopic mineralization has been studied. Compared to the general population, SFs have increased calcifications of the coronary arteries (8), carotid arteries (13), splenic artery $(10)$, and abdominal aorta $(9,10)$. However, prior to the current study, a comprehensive evaluation of biominerals in SFs had not been completed. Results of this study provide evidence that there exists a strong association between nephrolithiasis and pathologic mineral deposition at non-renal non-osseous sites, independent from common risk factors for atherosclerosis.

Consistent with published literature, the SFs in the current study had higher BMI and incidence of HTN, HLD, DM and smoking compared NSFs of equivalent age and gender. These comorbidities are independently linked to atherosclerosis and nephrolithiasis, making them possible contributors of biomineral deposition $(1-7,15,16)$. Increased age, HLD, male gender, and history of nephrolithiasis were significantly and independently associated with increased ectopic mineralization burden among all patients. Surprisingly, there was no significant relationship between increased ectopic mineral deposition and HTN, DM, or smoking. This may be attributable to the sample size of the study.

In the ultimate analysis comparing biominerals in SFs versus NSFs, the two groups were matched for atherosclerotic risk factors: BMI, HTN, HLD, DM, smoking history, gender, and hyperparathyroidism. Patients with kidney stone disease had significantly increased mineralization at the abdominal aorta, iliac arteries, prostate, mesentery, spleen, and pancreas compared to NSFs. Mineralization overall, across all anatomic sites studied, was also higher in SFs compared to NSFs (Figure 3). Pathologic mineralization deposition is also more pronounced in SFs without history of HTN, DM, HLD, hyperparathyroidism or smoking compared to NSFs without these medical conditions (Table S2). The increased systemic mineralization in SFs is independent of and appears to precede development of common causes of atherosclerosis.

A thorough understanding of the results of this study requires a review of the latest developments in the study of pathologic biomineral deposition. Nephrolithiasis and calcium-based vascular disease occur within a broad spectrum of pathologic human biomineralization. Under physiologic conditions, the skeleton, teeth, and otoconia are mineralized under a tightly regulated interplay between of intra- and extra-cellular signaling pathways and developmental gene networks (17). However, each of these pathways contain various sites for mutations to occur resulting in pathologic mineral formation. Pathologic or ectopic mineralization also can result from soft tissue injury or an imbalance of systemic concentrations of inhibitors and promoters of mineralization (18).

Nephrolithiasis and atherosclerosis are examples of pathologic ectopic mineralization. Known etiologies of pathologic mineralization include inflammation, oxidative stress, ionic disorders (hyperphosphatemia or hypercalcemia), and imbalance of promoters and inhibitors of mineralization (19). Pathologic biomineral deposition is also common in neoplastic processes and inflammatory adenopathy (20), and some etiologies of ectopic minerals are specific to particular anatomic regions. For example, pancreatic minerals can develop secondary to chronic 
pancreatitis (21), prostatic mineralization can result from stasis of prostatic fluids (22), and phleboliths are calcified thrombi in pelvic veins resulting from damaged vessel walls (23). Overall, the common link between the different etiologies of ectopic biomineral deposition is tissue damage resulting from an upstream event, inflammation (19-23).

Pathologic biominerals are present in both SFs and NSFs, yet we show that SFs have a propensity for greater pathologic biomineralization throughout the body. The underlying cause of this difference in systemically identified ectopic minerals between SFs and NSFs is currently unknown but likely holds crucial insights regarding formation of pathologic biominerals including kidney stones. Future studies will be directed toward identifying the elusive factors that contribute to increased biomineralization and kidney stone formation amongst SFs. Plausible candidates that could serve as factors for biomineralization include the imbalance between systemically active inhibitors and promoters. A recent genome-wide association study comparing SFs and NSFs identified sequence variants in several genes involving calcium-phosphate metabolism (24). Pyrophosphate is a known inhibitor of biomineralization (25), and alkaline phosphatase tightly regulates serum concentrations of pyrophosphate (26). Variations in concentrations and activities of these proteins may be responsible for the systemic propensity for mineralization in SFs and should therefore be compared in SFs and NSFs.

In addition to the implications regarding pathogenesis of nephrolithiasis, the findings of the current study have implications for public health. Presence of biominerals at non-renal anatomic sites may have major implications on the future health and wellbeing of patients. Aortic mineralization, for example, has been shown to associate with a six-fold increase in risk of cardiovascular death in middle-aged men, independent of other major cardiovascular risk factors (27). Many kidney stone patients do not have established diagnoses of extra-renal vascular disease at the time of initial presentation. The urologist may be the first to see these findings on CT scans performed for stone evaluation/surveillance. In essence, kidney stone formation may be a harbinger for poor longterm patient outcomes, especially for those patients with extensive vascular disease. Just as erectile dysfunction is a strong predictor of underlying or subsequent cardiovascular disease (28), nephrolithiasis, another organ-specific condition associated with cardiovascular risk factors and atherosclerosis, might be an early clinical predictor of cardiovascular events. Further research will be required to fully assess the utility of incorporating a history of nephrolithiasis and CT scan findings into risk calculations for cardiovascular events and stone recurrence.

This study has several limitations. Primarily, this is a retrospective study, so the directionality of cause and effect between kidney stone formation, systemic biomineralization, and atherosclerotic risk factors cannot be determined. Additionally, the most definitive method to compare systemic and site-specific mineralization would be to measure the exact volume of mineralization of each plaque identified. However, manual calculation of plaque volume was not feasible given the irregular shapes and multifocal nature of minerals at each site and the limitations of the software that is generally used in a patient-care CT. Although a radiologist was not responsible for reviewing CT scans, accuracy was maintained by adherence to the grading system described, and internal consistency was achieved by grading all CT scans by the same evaluator (BS). Whereas this study grouped all SF patients together, future studies that include more patients should stratify the differences in systemic mineralization among SFs with different stone types. Additionally, comorbidities were considered binary. Stratifying comorbidities by severity, such as by pack years among smokers or by glucose control amongst diabetics, would allow for more accurate matching. Finally, to assess whether nephrolithiasis correlates with increased systemic mineralization, a control group with CT scans readily available was necessary. Given the ethical barriers to prospectively imaging healthy patients, renal transplant donors were the most appropriate comparison group despite representing a community that may be healthier than other NSFs.

Going forward, we hope that this data can be used as evidence to support further research into the relationship between ectopic mineralization within the kidney and elsewhere in the body. Although this relationship remains poorly defined, this proof-of-concept study shows that SFs are likely to have a greater burden of systemic mineralization and cardiovascular comorbidities compared to the general population. The directionality of the relationship between vascular disease, kidney stone formation, and ectopic biomineralization should be studied to further our understanding of kidney stone pathogenesis and optimize preventative care.

\section{Acknowledgments}

Funding: None. 


\section{Footnote}

Reporting Checklist: The authors have completed the STROBE reporting checklist. Available at http://dx.doi. org/10.21037/tau-19-927

Conflicts of Interest: All authors have completed the ICMJE uniform disclosure form (available at http://dx.doi. org/10.21037/tau-19-927). MLS is owner and board member at Applaud Medical, Inc and owner of Ravine Group. He is a consultant and lecturer at C.R. Bard, Inc. and Boston Scientific. The other authors have no conflicts of interest to declare.

Ethical Statement: The authors are accountable for all aspects of the work in ensuring that questions related to the accuracy or integrity of any part of the work are appropriately investigated and resolved. Institutional review board (IRB) approval was obtained to review CT scans of previously established SF and renal donor databases (IRB\# 16-21330), and the study was conformed to the provisions of the Declaration of Helsinki (as revised in 2013). All patients provided a consent to review de-identified images and medical records for research purposes.

Open Access Statement: This is an Open Access article distributed in accordance with the Creative Commons Attribution-NonCommercial-NoDerivs 4.0 International License (CC BY-NC-ND 4.0), which permits the noncommercial replication and distribution of the article with the strict proviso that no changes or edits are made and the original work is properly cited (including links to both the formal publication through the relevant DOI and the license). See: https://creativecommons.org/licenses/by-nc-nd/4.0/.

\section{References}

1. Burke GL, Bertoni AG, Shea S, et al. The Impact of Obesity on Cardiovascular Disease Risk Factors and Subclinical Vascular Disease: The Multi-Ethnic Study of Atherosclerosis. Arch Intern Med 2008;168:928-35.

2. Curhan GC, Willett WC, Rimm EB, et al. Body size and risk of kidney stones. J Am Soc Nephrol 1998;9:1645-52.

3. Taylor EN, Stampfer MJ, Curhan GC. Obesity, Weight Gain, and the Risk of Kidney Stones. JAMA 2005;293:455-62.

4. Scales CD, Smith AC, Hanley JM, et al. Urologic Diseases in America Project. Prevalence of Kidney Stones in the
United States. Eur Urol 2012;62:160-5.

5. Kannel WB, McGee DL. Diabetes and Cardiovascular Disease: The Framingham Study. JAMA 1979;241:2035-8.

6. Kannel WB. Blood Pressure as a Cardiovascular Risk Factor: Prevention and Treatment. JAMA 1996;275:1571-6.

7. Shang W, Li Y, Ren Y, et al. Nephrolithiasis and risk of hypertension: a meta-analysis of observational studies. BMC Nephrol 2017;18:344-6.

8. Hsi RS, Spieker AJ, Stoller ML, et al. Coronary Artery Calcium Score and Association with Recurrent Nephrolithiasis: The Multi-Ethnic Study of Atherosclerosis. J Urol 2016;195:971-6.

9. Shavit L, Girfoglio D, Vijay V, et al. Vascular calcification and bone mineral density in recurrent kidney stone formers. Clin J Am Soc Nephrol 2015;10:278-85.

10. Stern KL, Ward RD, Li J, et al. Nonrenal Systemic Arterial Calcification Predicts the Formation of Kidney Stones. J Endourol 2019;33:1032-4.

11. Ferraro PM, Taylor EN, Eisner BH, et al. History of kidney stones and the risk of coronary heart disease. JAMA 2013;310:408-15.

12. Rule AD, Roger VL, Melton LJ, et al. Kidney Stones Associate with Increased Risk for Myocardial Infarction. J Am Soc Nephrol 2010;21:1641-4.

13. Reiner AP, Kahn A, Eisner BH, et al. Kidney stones and subclinical atherosclerosis in young adults: the CARDIA study. J Urol 2011;185:920-5.

14. Lin SY, Lin CL, Chang YJ, et al. Association Between Kidney Stones and Risk of Stroke: A Nationwide Population-Based Cohort Study. Medicine (Baltimore) 2016;95:e2847.

15. Tamadon MR, Nassaji M, Ghorbani R. Cigarette smoking and nephrolitiasis in adult individuals. Nephrourol Mon 2013;5:702-5.

16. Howard G, Wagenknecht LE, Burke GL, et al. Cigarette Smoking and Progression of Atherosclerosis: The Atherosclerosis Risk in Communities (ARIC) Study. JAMA 1998;279:119-24.

17. Kawasaki K, Buchanan AV, Weiss KM. Biomineralization in humans: making the hard choices in life. Annu Rev Genet 2009;43:119-42.

18. Giachelli CM. Vascular calcification mechanisms. J Am Soc Nephrol 2004;15:2959-64.

19. Evrard S, Delanaye P, Delanaye P, et al. Vascular calcification: from pathophysiology to biomarkers. Clin Chim Acta 2015;438:401-14.

20. Sheth S, Horton KM, Garland MR, et al. Mesenteric neoplasms: CT appearances of primary and secondary 
tumors and differential diagnosis. Radiographics 2003;23:457-73; quiz 535-6.

21. Lesniak RJ, Hohenwalter MD, Taylor AJ. Spectrum of causes of pancreatic calcifications. AJR Am J Roentgenol 2002;178:79-86.

22. Klimas R, Bennett B, Gardner WA Jr. Prostatic calculi: a review. Prostate 1985;7:91-6.

23. Luk AC, Cleaveland P, Olson L, et al. Pelvic Phlebolith: A Trivial Pursuit for the Urologist? J Endourol 2017;31:342-7.

24. Oddsson A, Sulem P, Helgason H, et al. Common and rare variants associated with kidney stones and biochemical traits. Nat Commun 2015;6:7975.

Cite this article as: Fernandez AM, Sherer BA, Gansky SA, Mena JD, Srirangapatanam S, Wiener SV, Chi T, Ho SP, Stoller ML. Ectopic biomineralization in kidney stone formers compared to non-stone formers. Transl Androl Urol 2020;9(5):2129-2137. doi:10.21037/tau-19-927
25. Moochhala SH, Sayer JA, Carr G, et al. Renal calcium stones: insights from the control of bone mineralization. Exp Physiol 2008;93:43-9.

26. Lomashvili KA, Garg P, Narisawa S, et al. Upregulation of alkaline phosphatase and pyrophosphate hydrolysis: Potential mechanism for uremic vascular calcification. Kid-ney Int 2008;73:1024-30.

27. Witteman JCM, Witteman JM, Kok F, et al. Aortic calcification as a predictor of cardi-ovascular mortality. Lancet 1986;2:1120-2.

28. Thompson IM, Tangen CM, Goodman PJ, et al. Erectile Dysfunction and Subsequent Cardiovascular Disease. JAMA 2005;294:2996-3002. 
Supplementary

Table S1 Patient characteristics among participants with no history of DM, HTN, HLD, HPTH or smoking

\begin{tabular}{lccc}
\hline & Stone formers $(\mathrm{n}=77)$ & Non-stone formers $(\mathrm{n}=85)$ & $\mathrm{P}$ value \\
\hline Age at CT (mean \pm SD) & $43.6 \pm 11.9$ & $47.4 \pm 7.6$ & 0.02 \\
Body mass index (mean \pm SD) & $27.4 \pm 7$ & $27.4 \pm 3.9$ & 0.96 \\
Male & 32 & 37 & 0.8 \\
Female & 45 & 48 & 0.8 \\
\hline
\end{tabular}

Table S2 Odds ratios of mineralization severity scores in SFs and NSFs without history of DM, HTN, HLD, HPTH or smoking

\begin{tabular}{|c|c|c|}
\hline & OR & $\mathrm{P}$ \\
\hline Iliac arteries & 3.34 & $<0.01$ \\
\hline Pelvic veins & 0.88 & 0.66 \\
\hline Prostate & 0.36 & $<0.01$ \\
\hline Mesentery/bowel & 8.7 & $<0.01$ \\
\hline Spleen & 1.46 & 0.69 \\
\hline Pancreas & 1.04 & 0.15 \\
\hline Overall & 1.04 & 0.16 \\
\hline
\end{tabular}

